\title{
EFFICIENT PROTOCOLS FOR COMPUTING THE OPTIMAL SWAP EDGES OF A SHORTEST PATH TREE
}

\author{
Paola Flocchini, ${ }^{1}$ Antonio Mesa Enriques, ${ }^{2}$ Linda Pagli, ${ }^{3}$ Giuseppe Prencipe, ${ }^{3}$ \\ and Nicola Santoro ${ }^{4}$ \\ ${ }^{1}$ University of Ottawa, Canada, ${ }^{2}$ Universidad de la Habana, Cuba, ${ }^{3}$ Università di Pisa, \\ Italy, ${ }^{4}$ Carleton University, Canada
}

\begin{abstract}
We consider the problem of computing the optimal swap edges of a shortest-path tree. This theoretical problem arises in practice in systems that offer point-offailure shortest-path rerouting service in presence of a single link failure: if the shortest path is not affected by the failed link, then the message will be delivered through that path; otherwise, the system will guarantee that, when the message reaches the node where the failure has occurred, the message will then be rerouted through the shortest-path to its destination. There exist highly efficient serial solutions for the problem, but unfortunately because of the structures they use, there is no known (nor foreseeable) efficient distributed implementation for them. A distributed protocol exists only for finding swap edges, not necessarily optimal ones.

We present two simple and efficient distributed algorithms for computing the optimal swap edges of a shortest-path tree. One algorithm uses messages containing a constant amount of information, while the other is tailored for systems that allow long messages. The amount of data transferred by the protocols is the same and depends on on the structure of the shortest-path spanning-tree; it is no more, and sometimes significantly less, than the cost of constructing the shortest-path tree.
\end{abstract}

Keywords: Fault-Tolerant Routing, Point of Failure Rerouting, Shortest Path Spanning Tree, Weighted Graphs, Distributed Algorithms, Data Complexity

\section{Introduction}

Consider an undirected graph $G=(V, E)$ with weighted edges. Let $T_{r}$ be the shortest-path spanning-tree of $G$ rooted in $r \in V$. The removal of any edge $e$ of $T_{r}$ will disconnect $T_{r}$ into two subtrees. If $G$ is biconnected, there will always be at least an edge $e^{\prime} \in E(G) \backslash E\left(T_{r}\right)$ that would join the two disconnected subtrees forming a new spanning tree $T^{\prime}$ of $G$. Any such an edge is called a swap edge for $\boldsymbol{e}$; let $\boldsymbol{S}_{\boldsymbol{r}}(\boldsymbol{e})$ denote the set of swap edges for $\boldsymbol{e}$. An optimal swap edge (or bridge) for $\boldsymbol{e}=(u, v)$ is any swap edge for $\boldsymbol{e}$ such that the distance from $u$ to the source $\boldsymbol{r}$ in the new tree 
$T^{\prime}$ is minimized; more precisely, the optimal swap edge for $e=(u, v)$ is the swap edge $e^{\prime}=\left(u^{\prime}, v^{\prime}\right) \in S_{r}(e)$ such that $d_{T^{\prime}}(u, r)=d_{T_{r}}\left(u, u^{\prime}\right)+\left|\left(u^{\prime}, v^{\prime}\right)\right|+d_{T_{r}}\left(v^{\prime}, r\right)$ is minimum.

The (optimal) swap edges problem for a shortest-path tree $T(r)$ is the one of determining for each edge in $T_{r}$ an (optimal) swap edge. We are interested in the distributed solution of the optimal swap edge problem.

\section{The Framework and Previous Work}

This problem arises in the context of static fault-tolerant routing; specifically, it arises within the larger problem of augmenting the information of the shortest-path routing tables so to make them operate in spite of the fact that, at any time, one link (not necessarily the same at all time) might be down.

Consider a network where the routing tables contain information enabling shortestpath routing between any two nodes in the network. In this context, if one wants to continue to offer shortest-path routing after the failure of an arbitrary single link, a service called shortest-path rerouting $(S R)$, the amount of additional information that needs to be stored in the tables might be formidable. This is because the failure of a single edge can dramatically change all the shortest-path information.

To reduce the amount of communication and of storage, a simple and convenient alternative is to offer, after the failure of an arbitrary single link, a lower quality service called point-of-failure rerouting $(P R)$ : if the shortest path is not affected by the failed link, then the message will be delivered through that path; otherwise, the system will guarantee that, when the message reaches the node where the failure has occurred (the "point of failure"), the message will then be re-routed to its destination. This approach has clearly the advantage that there is no need to broadcast a link failure and its subsequent reactivation (if any).

The amount of storage of the $P R$ approach will depend on what type of information is being kept at the nodes to do the rerouting.

An effi cient solution has been recently presented. In fact Ito et al [6] showed that the amount of storage can be reduced by precomputing for each possible link failure a swap edge to replace the failed one, for every possible destination, and storing this information in the tables. In particular, for each destination, a node stores only one link in addition to the one in the fault-free shortest-path [6]. Its implementation requires to solve $\boldsymbol{n}$ instances of the swap edges problem, one for each choice of $s \in V$. A sequential algorithm to solve the swap edge problem has been presented [6]; this algorithm can possibly be efficiently implemented in a distributed setting.

A basic drawback of the point-of-failure rerouting service is that, if the failure occurs, the system does not make any guarantee other than message delivery. Although acceptable in some contexts, this level of service might not be tolerable in general. On the other hand, the shortest-path rerouting service that always guarantee optimal routing, is too costly to implement. In a sense, $S R$ and $P R$ are two extreme approaches.

In between there two extreme, there is another type of service, the point-of-failure shortest-path rerouting (PSR), that has some of the service quality of $S R$ while keeping all the space and communication advantages of $P R$. In $P S R$, if the shortest path is not affected by the failed link, then the message will be delivered through that path; otherwise, the system will guarantee that, when the message reaches the node where 
the failure has occurred, the message will then be re-routed through the shortest-path to its destination. This improved quality of service can be achieved by precomputing for each possible link failure a optimal swap edge, to replace the failed one, for each possible destination, and storing this information in the tables. In particular, it is enough for a node just to know (how to reach) an optimal swap edge for each possible destination. In other words, $P S R$ can be achieved with the same amount of storage as $P R$ : for each destination, a node stores only one link in addition to the one in the fault-free shortest-path.

For its implementation, $P S R$ requires the solution of $\boldsymbol{n}$ instances of the more difficult optimal swap edgesproblem, one for each choice of $\boldsymbol{s} \in V$.

The problem of computing all the optimal swap edges for a shortest-path tree has been attacked by Nardelli, Proietti, and Widmayer [8]. They showed that the problem can be solved sequentially in $O(m \cdot \alpha(m, n))$ time, where $\alpha(m, n)$ is the functional inverse of Ackermann's function. This bound is achieved using Tarjan's sophisticated technique for union-find, which requires the construction of transmuters [10]. Unfortunately, there is currently no efficient distributed implementation of this sequential technique; since in a distributed network setting the construction of transmuters requires complete global network information at some node, it is doubtful whether this approach will become feasible at all.

Summarizing, optimal swap edges problem is both an interesting graph-theoretic problem on its own (studied as such in [8]) and a crucial component to implement a point-of-failure shortest-path rerouting strategy (described in [6]). Currently, there is no distributed solution (in [6], the problem is posed but no solution given). Clearly any such a solution should not add significantly to the overall cost of constructing the final routing tables. In particular, the computation should not require more messages (at least in order of magnitude) than those used to construct the shortest-path tree.

\section{Our Contribution}

In this paper we present an efficient distributed solution to the optimal swap edges problem.

Given a shortest-path spanning-tree $T_{r}$, the proposed protocol determines at each node $\boldsymbol{x}$ the optimal swap edge for $\boldsymbol{e}_{\boldsymbol{x}}$. The algorithm uses $\boldsymbol{O}\left(n_{r}^{*}\right)$ messages of constant size, where $n_{r}^{*}$ is the size of the transitive closure of $\boldsymbol{T}_{r} \backslash\{r\}$; observe that $0 \leq n_{r}^{*} \leq$ $(n-1)(n-2) / 2$.

If longer messages are allowed, the same strategy can be modified to construct a different algorithm that uses only $\boldsymbol{O}(n)$ such messages.

Providing a uniform comparison between protocols using different sized messages, the data complexity of a protocol measures the total amount of data exchanged during the execution; in our context, a node, an edge, a label, a weight, and a distance are each a unit of data. Both algorithms have an overall data complexity of $O\left(n_{r}^{*}\right)$.

Notice that this cost is always less, and oftentimes substantially so, than the cost of constructing a shortest-path spanning-tree. We actually conjecture that such a cost is optimal.

Further notice that the information assumed available by our algorithms can be acquired during the shortest-path spanning-tree construction, without increasing the order of magnitude of the message and information complexity of that process. Should 
this information not be provided, it can be easily acquired with an $O(m)$ data complexity.

The paper is organized as follows. In the next section we introduce some definitions and terminology. The new distributed algorithm for constructing all the optimal swap edges for a given shortest-path spanning-tree using constant-size messages is described and analyzed in Section 3. In Section 4, we present a more efficient algorithm for systems allowing long messages. The concluding remarks and open problems are in Section 6.

\section{Definitions and Terminology}

Let $G=(V, E)$ be a simple undirected graph, with $n=|V|$ vertices and $m=|E|$ edges. A subgraph $G^{\prime}=\left(V^{\prime}, E^{\prime}\right)$ of $G$ is any graph where $V^{\prime} \subseteq V$ and $E^{\prime} \subseteq E$. If $V^{\prime} \equiv V, G^{\prime}$ is a spanning subgraph. A path $P=\left(V_{p}, E_{p}\right)$ is a subgraph of $G$, such that $V_{p}=\left\{v_{1}, \ldots, v_{s}\right\} \mid v_{i} \neq v_{j}$, for $i \neq j$, and $\left(v_{i}, v_{i+1}\right) \in E_{p}$, for $1 \leq i \leq s-1$. If $\boldsymbol{v}_{1}=\boldsymbol{v}_{\boldsymbol{s}}$ then $P$ is a cycle. A graph $G$ is connected if, for each pair $\left\{\boldsymbol{v}_{\boldsymbol{i}}, \boldsymbol{v}_{j}\right\}$ of its vertices, there exists a path connecting them. A graph $G$ is biconnected if, after the removal of anyone of its edges it remains connected. A tree is a connected graph with no cycles.

A non negative real value called weight (or length) and denoted by $|\boldsymbol{e}|$ is associated to each edge $\boldsymbol{e}$ in $G$. Given a path $P$, the length of the path is the sum of the lengths of its edges. The distance $d_{G^{\prime}}(x, y)$ between two vertices $x$ and $y$ in a connected subgraph $G^{\prime}$ of G, is the length of the shortest path from $x$ to $y$ in $G^{\prime}$. For simplicity, in the following we will denote $d_{G}(x, y)$ simply by $d(x, y)$.

For a given vertex $r$, called source, the shortest path tree (SPT) of $r$ is the spanning tree $T_{r}$ rooted at $r$ such that the path in $T_{r}$ from $r$ to any node $v$ is the shortest possible one; i.e., $\forall x \in V d_{T_{r}}(x, r)=d(x, r)$.

The removal of any edge $\boldsymbol{e}$ of $\boldsymbol{T}_{\boldsymbol{r}}$ will disconnect $T_{\boldsymbol{r}}$ into two subtrees. If $G$ is biconnected, there will always be at least an edge $e^{\prime} \in E(G) \backslash E\left(T_{r}\right)$ that will join the two disconnected subtrees forming a new spanning tree $T^{\prime}$ of $G$. Any such an edge is called a swap edge for $\boldsymbol{e}$; let $\boldsymbol{S}_{r}(\boldsymbol{e})$ denote the set of swap edges for $\boldsymbol{e}$.

An optimal swap edge (or bridge) for $\boldsymbol{e}=(u, v)$ is any swap edge for $\boldsymbol{e}$ such that the distance from $u$ to the source $r$ in the new tree $T^{\prime}$ is minimized; more precisely, an optimal swap edge for $e=(u, v)$ is a swap edge $e^{\prime}=\left(u^{\prime}, v^{\prime}\right) \in S_{r}(e)$ such that $d_{T^{\prime}}(u, r)=d_{T_{r}}\left(u, u^{\prime}\right)+\left|\left(u^{\prime}, v^{\prime}\right)\right|+d_{T_{r}}\left(v^{\prime}, r\right)$ is minimum. As an example, consider the biconnected weighted graph $G$ shown in Figure (1.a), and the shortestpath spanning tree $T_{A}$ rooted shown in Figure in (1.b). It is easy to verify that the optimal swap edge for $(C, A)$ is $(F, B)$.

The optimal swap edgesproblem for $T(r)$ is the problem of determining an optimal swap edge for each edge in $T_{r}$.

As already mentioned, a sequential algorithm solving this problem was given in [8]. We are interested in the distributed solution of the optimal swap edge problem. We consider a distributed computing system with communication topology G. Each computational entity $\boldsymbol{x}$ is located at a node of $G$, has local processing and storage capabilities, has a distinct label $\lambda_{x}(e)$ from a totally ordered set associated to each of its incident edges $\boldsymbol{e}$, knows the weight of its incident edges, and can communicate with its neighboring entities by transmission of bounded sequence of bits called messages. 


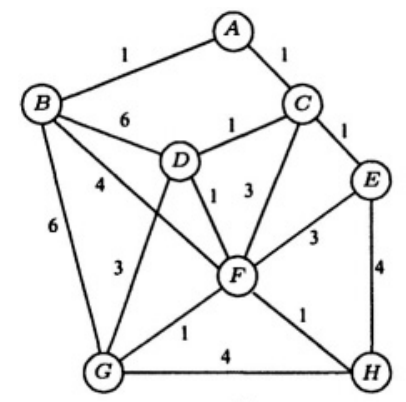

(a)

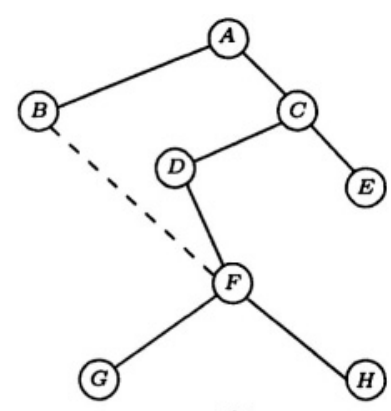

(b)

Figure l. (a) A biconnected weighted graph $G$. (b) The shortest-path spanning tree $T_{A}$ rooted in $A$; the dotted edge $(F, B)$ is the optimal swap edge for $(C, A)$.

The communication time includes processing, queueing, and transmission delays, and it is fi nite but otherwise unpredictable. In other words, the system is asynchronous. All the entities execute the same set of rules, called distributed algorithm.

In the following, when no ambiguity arises, we will use the terms entity, node and vertex as equivalent; analogously, we will use the terms link, arc and edge interchangeably.

\section{Computing All Optimal Swap Edges}

We now present a solution to the problem of distributively computing all optimal swap edges for a given shortest-path spanning tree $T_{r}$.

\section{Basic Properties and Tools}

In our algorithm we make use of some known properties of rooted trees. In $T_{r}$ each node except the root $r$ has a unique parent, and each edge connects a node to its parent.

PROPERTY 1 The partial order induced by the relation parent has dimension at most 2.

Consider in fact the labelling $\alpha: V \rightarrow\{1, \ldots, n\}^{2}$ defined as follows. Given $\mathcal{T}_{r}$, for $x \in V$ let $\alpha(x)=(a, b)$, where $a$ is the numbering of $x$ in the preorder traversal ${ }^{1}$ of $T_{r}$; and $b$ is the numbering of $\boldsymbol{x}$ in the inverted preorder traversal of $T_{r}$, i.e., when the order of the visit of the children is inverted. The labels associated to the nodes in the tree of Figure 1.b are shown in Figure 2.a.

Let $T_{r}[x]$ denote the subtree of $T_{r}$ rooted in $x$. Any node $y$ in the subtree $T_{r}[x]$ is said to be a descendant of $x$. Let $\operatorname{Desc}_{r}(x)$ be the set of the descendants of $x$ in $T_{r}$; note that, bydefinition, $x \in \operatorname{Desc}(x)$. Interestingly, the lexicographic order $\succ$ between the labels assigned by $\alpha$ completely characterizes the descendant relationship in a rooted tree:

\footnotetext{
${ }^{1}$ Since the labelling of the incident links is drawn from a totally ordered set, this numbering is unique.
} 
PROPERTY 2 A node $y$ is descendant of a node $x$ in $T_{r}$ if and only if $\alpha(y) \succeq \alpha(x)$.

Property 2 can be easily verifi ed and it is known as a folklore method to check relationships among nodes in trees.

Furthermore, there exists a simple relationship between swap edges and the descendants.

PROPERTY 3 An edge $(u, v) \in E \backslash E\left(T_{s}\right)$ is a swap for $e_{x} \in E\left(T_{s}\right)$ if and only if only one of $\boldsymbol{u}$ and $\boldsymbol{v}$ (but not both) is in Desc $\boldsymbol{r}(\boldsymbol{x})$.

For brevity, we will denote the set $S_{r}\left(e_{x}\right)$ of all swap edges for $e_{x}$ simply by $S(x)$, and by $\operatorname{In} S(x) \subseteq S(x)$ the set of those that are incident on $x$. The last useful property states that the swap edges for $\boldsymbol{e}_{\boldsymbol{x}}$ consists only of all the swap edges incident to $\boldsymbol{x}$ and to its descendants.

\section{PROPERTY 4 For all $x \in V S(x)=\bigcup_{y \in\left(\operatorname{Desc}_{r}(x)\right.} \operatorname{In} S(y)$.}

Properties 2,3, and 4 provide a powerful computational tool for determining which edges are possible candidate for being optimal swap edges. We will now see how to effi ciently use this tool.

\section{The Algorithm}

By definition, a node $x$ knows the weight of all its incident links, and can distinguish those that are part of $T_{s}$ from those that are not; of those that are part of $T_{s}, x$ can distinguish the one that leads to its parent from those leading to its children.

We assume that each node $\boldsymbol{x}$ knows its distance from $r$, the distances of its neighbors from $r$, its own pair $\alpha(x)$, as well the pairs of its neighbors. If not available, this information can be easily and efficiently acquired.

In the proposed algorithm, each node $x$ computes an optimal swap edge for $\boldsymbol{e}_{\boldsymbol{x}}$, i.e., the swap edge for $\boldsymbol{e}_{\boldsymbol{x}}$ in the shortest path from $x$ to $r$ in $E \backslash\left\{\boldsymbol{e}_{\boldsymbol{x}}\right\}$. We shall denote such an edge as $\boldsymbol{b}_{\boldsymbol{x}}$ and call it the bridge of $\boldsymbol{x}$. A node $\boldsymbol{x}$ also contributes, if necessary, to the computation of the bridges of other nodes.

\section{Computing its bridge.}

To compute its bridge $b_{x}$, a node $x$ :

1 It determines which of its incident edges are swaps for $e_{x}$; i.e., it constructs the set $\operatorname{In} S(x)$. It then sets $L(x)=\operatorname{In} S(x)$.

2 If $x$ is not a leaf (and if it does not have the information already),

(a) it requests from each child $y$ a swap edge (if any) for $e_{x}$ that is incident on a descendent of $y$, and among all edges satisfying the above, the distance from $\boldsymbol{y}$ to $\boldsymbol{r}$ using this swap edge instead of $\boldsymbol{e}_{\boldsymbol{x}}$ is minimized.

(b) It waits until it receives a reply from all its children. It adds each received edge to the set $\boldsymbol{S}(\boldsymbol{x})$.

3 For each $e \in L(x), x$ computes its distance from $r$ using $\boldsymbol{e}$ instead of $\boldsymbol{e}_{\boldsymbol{x}}$ (i.e., in $\left.T_{r}-\left\{e_{x}\right\} \cup\{e\}\right)$. It then sorts $L(x)$ so that the corresponding distances are in 


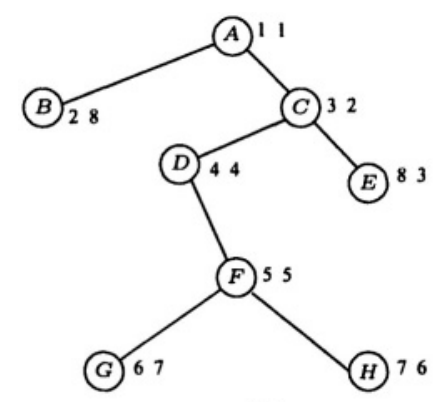

(a)

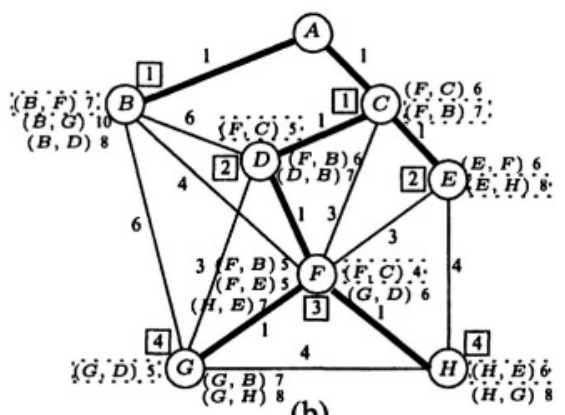

(b)

Figure 2. Computing all the optimal swap edges in the graph $G$ of figure 1 for the SPT rooted in $A$. (a) For each node $x$ there is shown $\alpha(x)$. (b) For each node $x$ there are shown: the distance of $x$ from the root (in the solid box): the elements of $L(x)$, among which $b_{x}$ (in the dotted box).

non-decreasing order. Let $\min _{x}$ be the smallest edge in the sorted $L(x)$; then $b_{x}=\min _{x}$.

Each node $x$ also contributes to the calculation of the bridge of its predecessors. It does so as following.

Cooperating with the other nodes.

1 As soon as its bridge $b_{x}$ has been computed, $x$ sends it to its parent.

2 If requested by its parent to find the best swap edge feasible with some edge $e$, then:

(a) It creates a set $L(x, e)$ by removing from $L(x)$ all the incident links that are not swap edges for $\boldsymbol{e}$;

(b) if the link in $L(x)$ sent by child $y$ is not a swap for $\boldsymbol{e}$, then $x$ removes it from $L(x, e)$,requests $y$ to send its best swap edge for $\boldsymbol{e}$, waits to receive such an edge (or notifi cation that none exists), and adds it to $L(x, e)$

(c) $x$ sorts $L(x, e)$ and sends the new $\min _{x}$ to its parent (it will send NIL if $L(x, e)=\emptyset)$.

Note that, because of Properties 2 and 3, to determine if an edge is a swap edge is sufficient to examine the relationship "descendant", which in turn is uniquely determined by the mapping $\alpha$. Hence, in the following, we shall use the term "feasible for $\alpha(x)$ " to mean "swap edge for $\boldsymbol{e}_{\boldsymbol{x}}$ " without any loss of precision.

Algorithm ALL BRIDGES, reported below, describes the state-event-action set of rules: it specifies what action must a node perform if in a given state.

Initially, all nodes are in state COMPUTING and start the execution. Each node $x$ maintains a list $L(x)$ of possible swap edges. Initially $L(x)$ contains all the links incident on $x$ that are not in the tree. 


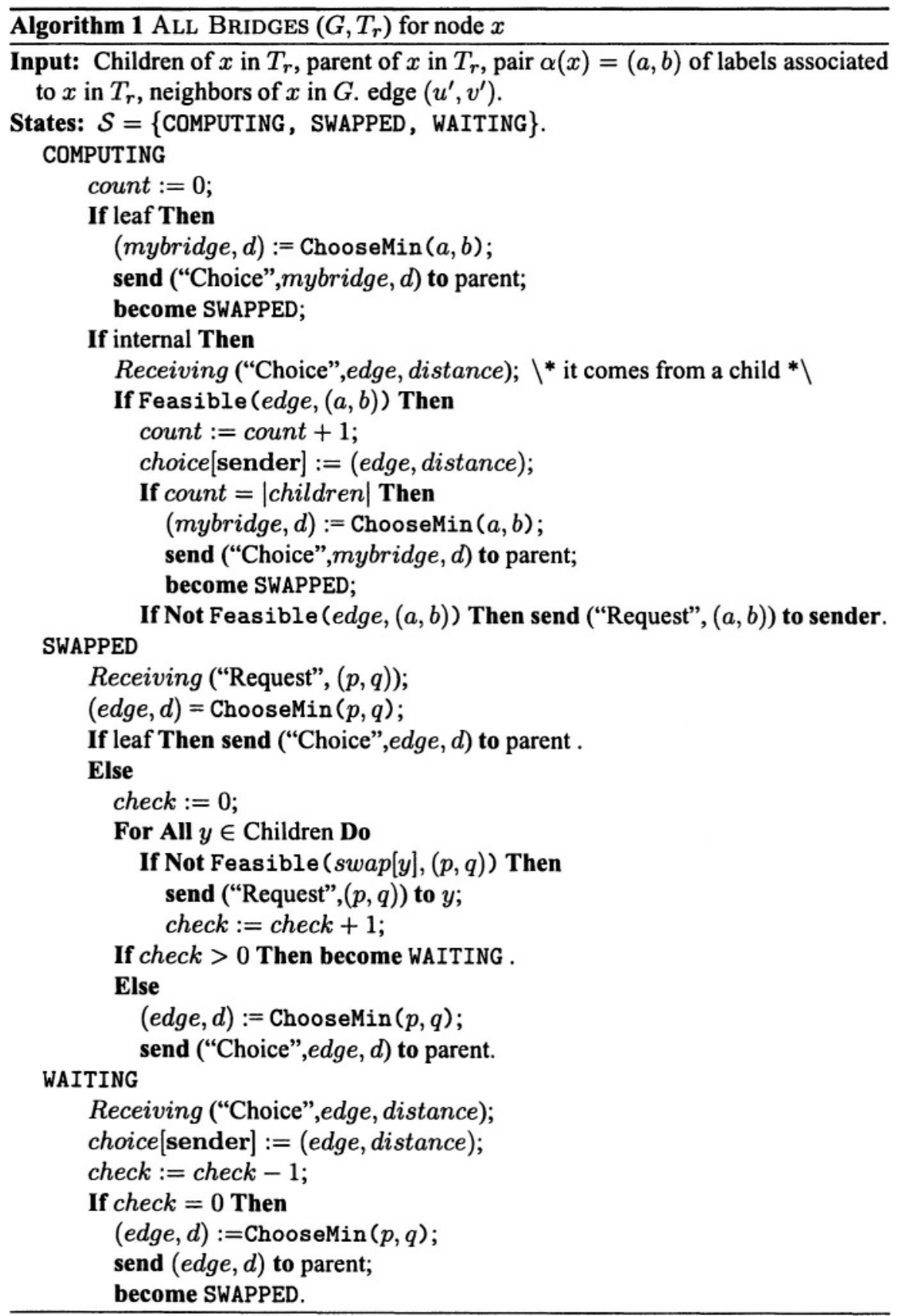


To each $e=(w, z) \in L(x)$, where $\boldsymbol{w}$ is a descendent of $x$ (possibly, $x=w$ ), there is associated the pair $\boldsymbol{\alpha}(\boldsymbol{z})$ as well as the distance

$$
d[e]=d(x, w)+|(w, z)|+d(z, r) .
$$

The set $L(x)$ is kept sorted w.r.t. the distances. Note that while each node knows its distance from the root, the distances $d[\boldsymbol{e}]$ must be computed. This can be easily done: when a swap edge $\boldsymbol{e}$ is transmitted by a child to a parent along $(u, v)$, together with a distance $\boldsymbol{d}$, node $\boldsymbol{v}$ will increment the distance by $|(u, v)|$.

Function Choosemin $(a, b)$ determines the swap edge with minimum distance in $L(x)$ that is feasible with $(a, b)$ (for the leaves all the swap edges are feasible), its output is $(\boldsymbol{e}, \boldsymbol{d})$, where $\boldsymbol{e}$ is such an edge and $\boldsymbol{d}$ is the distance between $\boldsymbol{x}$ and $\boldsymbol{r}$ using $\boldsymbol{e}$ as a swap edge. If no such an edge exists, function $\operatorname{ChooseMin}(\boldsymbol{a}, \boldsymbol{b})$ returns NIL.

The Boolean function Feasible (edge, $(a, b))$ determines whether $e$ dge is feasible with the pair of labels $(a, b)$; by definition, if $e d g e=N I L$, Feasible is always TRUE, regardless of $(a, b)$. Let $e=(z, w)$. The feasibility of the swap edge $e$ is checked by comparing the pair $(a, b)$ with the pair corresponding to $w$.

It is understood that when sending information about an edge $\boldsymbol{e}$, as in the "Choice" messages, this information include the pairs of labels associated to the end nodes of $e$.

Example. As an example consider the SPT of the graph of Figure 1, shown in Figure 2. According to the algorithm, the leaf nodes $B, G, H$, and $E$ compute their bridges directly and become SWAPPED. Node $F$ receives swap edges from $G$ and $H$ and can computes its bridge $[(F, C), 4]$ becoming SWAPPED. Node $D$ receives the swap edge $[(F, C), 5]$ from its only child $F$, and this becomes its bridge. Node $C$ instead receives non feasible edges from both $D$ and $E$; it then sends to both of them a request for a feasible edge. Node $E$ does not have edges feasible with $C$; hence it sends $N I L$. As for $D$, as the swap link it had received from $F$ is not feasible for $C$, it will forward the request to $\mathrm{F}$. Since the swap edge known to $F((F, C))$ is not feasible for $C, F$ forwards the request to the leaves $G$ and $H$. At this point there is a propagation of swap edges feasible with $C$. In fact, $G$ sends up [( $G, B), 7], H$ sends up NIL, $F$ chooses as minimum $[(F, B), 5]$ and returns this information to $D$ which sends it to $C$. Receiving $N I L$ from $E$ and $[(F, B), 6]$ from $D$, node $C$ can conclude its computation selecting $[(F, B), 7]$ as its bridge.

\section{Analysis}

The correctness of Algorithm ALL BRIDGES is established by the following Theorem.

\section{THEOREM 1 In algorithm ALL BRIDGES:}

(i) each node $\boldsymbol{u}$ correctly computes $\boldsymbol{b}_{\boldsymbol{u}}$;

(ii) if so requested by its parent, each node $\boldsymbol{u}$ will determine among the swap edges incident to its subtree and feasible with $\alpha(\boldsymbol{e})=(p, q)$, if any, one edge $\boldsymbol{e}^{\prime}$ that minimizes the distance between $u$ and $\boldsymbol{r}$ in $T_{r}-\{\boldsymbol{e}\} \cup\left\{\boldsymbol{e}^{\prime}\right\}$.

Proof Removal of $\boldsymbol{e}_{\boldsymbol{u}}$ partitions $\boldsymbol{T}_{\boldsymbol{r}}$ in two subtrees, one rooted in $\boldsymbol{r}$ the other in $\boldsymbol{u}$. By definition, any feasible swap edge, and hence $\boldsymbol{h}_{\boldsymbol{\iota}}$, must have an endpoint in each 
component. The proof will be by induction on the height $h(u)$ of the subtree of $T_{r}$ rooted in $u$.

Basis. $h(u)=0$; i.e., $u$ is a leaf. In this case, one components contains only $u$, while the other contains all the other nodes. In other words, the only possible swap edges are incident on $\boldsymbol{u}$. Thus, $\boldsymbol{u}$ correctly computes $\boldsymbol{b}_{\boldsymbol{u}}$, proving (i); it can also immediately determine the feasibility of any of those links with respect to any pair of labels, and thus answer correctly any received query, proving (ii).

Induction step. Let the theorem hold for all nodes $v$ with $k-1 \geq h(v) \geq 0$; we will now show that it holds for $u$ with $h(u)=k$. Since $u$ is not a leaf, the subtree $T_{r}[u]$ rooted at $u$ contains at least two nodes. Consider the set $S(u)$ of all feasible swap edges for $e_{u}$; clearly, if $e=(w, z) \in S(u)$ then one of its end point, say $w$, is in $T_{r}[u]$ (and thus a descendent of $u$ ), while the other say $z$, is not.

Let $v$ be a child of $u$; then $h(v)<k$. It follows that, by inductive hypothesis, when asked by $u, v$ will send to $u$ the edge in $\operatorname{Swap}(v)$ that, among those feasible with $\boldsymbol{e}_{\boldsymbol{u}}$, minimizes the distance between $\boldsymbol{v}$ and $\boldsymbol{r}$. We will now show that this information is sufficient for $u$ to correctly determine its optimal swap edge $\boldsymbol{b}_{\boldsymbol{u}}$.

By definition of bridge, $b_{\iota}$ is the edge $e=(w, z)$ in $S(u)$ that minimizes the quantity $d_{u}[e]=d(u, w)+|(w, z)|+d(z, r)$. By Property 4 , the optimal swap edge is either incident on $u$ or on a strict descendent of $u$. Clearly $u$ can locally determine its distance from $\boldsymbol{r}$ for any of its incident swap edges, and determine the minimum one. If $\boldsymbol{e}$ is not incident on $\boldsymbol{u}$, it is in the subtree $T_{r}[v]$ rooted in a child $v$ of $u$; furthermore, $e$ is the swap edge in $S(v)$ that, among those feasible with $\boldsymbol{e}_{\boldsymbol{u}}$, minimizes the distance between $\boldsymbol{v}$ and $r$. In other words, once $\boldsymbol{u}$ obtains from each child $\boldsymbol{v}^{\prime}$ the swap edge $\boldsymbol{e}^{\prime} \in \boldsymbol{S}\left(\boldsymbol{v}^{\prime}\right)$ that, among those feasible with $\boldsymbol{e}_{\boldsymbol{u}}$, minimizes the distance between $\boldsymbol{v}_{\boldsymbol{i}}$ and $\boldsymbol{r}, \boldsymbol{u}$ can determine the minimum one. Since, by inductive hypothesis, every child of $u$ sends this information to $u$, it follows that $u$ can correctly determine its optimal swap edge, proving Part (i) of the Theorem.

To prove Part (ii), it is sufficient to observe that, by Property $3, u$ can determine which of its incident swap edges are feasible with a given pair $(p, q)$; furthermore, since the height of its children in $T_{r}$ is less than $k$, then by inductive hypothesis it can obtain from them the "best" swap edge in their subtree feasible with $(p, q)$. Therefore, $u$ can determine among the swap edges incident to its subtree and feasible with $(p, q)$, if any, one that minimizes the distance between $u$ and $r$, proving Part (ii).

Let us now examine the message complexity of the proposed algorithm. Let $n_{r}^{*}$ be the number of edges of the transitive closure of $T_{r} \backslash\{r\}$; observe that $0 \leq n^{*} \leq$ $(n-1)(n-2) / 2$. 
THEOREM 2 The message complexity of Algorithm ALL BRIDGES is at most $2 n^{*}+$ $n-1$.

Proof Each node, once computed its optimal swap edge, sends a message to its parent, for a total of $n-1$ "Choice" messages. To compute its optimal swap edge, a node $x$ might send a "Request" message to all its children (if the original information provided by them is not feasible), which in turn might send to their children (if no feasible information was received), and so on. Thus, in the worst case, each descendant of $x$ will receive a "Request" and reply a "Choice" for a total of $2|\operatorname{Desc}(x)|$ messages.

Since each node, except the root, must compute its optimal swap edge, this process will require at most

$$
\sum_{x \neq r} 2|\operatorname{Desc}(x)|=\sum_{x} 2(|\operatorname{Ance}(x)|-1)=2 n_{r}^{*},
$$

where Ance $(\boldsymbol{x})$ denotes the set of ancestors of $\boldsymbol{x}$.

A node, an edge, a label, a weight, and a distance are all unit of data. To evaluate the overall data complexity of the algorithm we need to consider the message size; since each message contains only a constant number of units of information, we have:

THEOREM 3 The data complexity of the distributed Algorithm ALL BRIDGES is $O\left(n_{r}^{*}\right)$.

Observe that the data complexity needed by our algorithm to compute all the optimal swap edges of a shortest-path spanning-tree is no more (and very often dramatically less) than the one of computing the shortest-path spanning-tree itself $[3,4,2]$.

\section{An O(n) Messages Algorithm}

In this section, we discuss how the algorithm of Section 3 can be modified in order to reduce the message complexity to $O(n)$ in case that longer messages are allowed. The overall information complexity of the new algorithm remains of $\boldsymbol{O}\left(n_{r}^{*}\right)$.

The idea is now that each node simultaneously computes the "best" feasible swap edges, not only for itself, but also for all its ancestors in the SPT. The modifi ed algorithm will be described only at high level. It consists simply of a broadcast phase started by the children of the root, followed by a convergecast phase started by the leaves.

\section{THEOREM 4 Each node $u \neq r$ :}

(i) correctlycomputes $\boldsymbol{b}_{\boldsymbol{u}}$;

(ii) determinesfor each ancestor $v \neq r$ the best swap edgefeasible with $\alpha(v)$, if any.

Proof First observe that, as a result of the broadcast, every node will receive the pair associated to each of its ancestors (except $r$ ); hence it can determine feasibility, for 


$\overline{\text { Algorithm 2 ALL BRIDGES-2 }}$

1 Each child $x$ of the root starts the broadcast by sending a list containing $\alpha(x)$ to its children.

2 Each node $y$, adds $\alpha(y)$ to the received list and sends it to its children.

\section{[Convergecast.]}

1 Each leaf $z$ first computes its own bridge. It then computes the best feasible swap edge for each of its ancestors, and sends the list of those edges to its parent (if different from $r$ ).

2 An internal node $y$ waits until it receives the list of best swap edges from each of its children. Based on the received information and on $\operatorname{In} S(y)$, it computes its bridge $b_{y}$. It also computes the best feasible swap edge for each of its ancestors, and sends the list of those edges to its parent (if different from $r$ ).

each ancestor, of any available set of swap edges. The proof is by induction on the height $h(u)$ of the subtree of $T_{r}$ rooted in $u$.

Basis. $h(u)=0$; i.e., $u$ is a leaf. In this case, one components contains only $u$, while the other contains all the other nodes. In other words, the only possible swap edges are incident on $u$. Thus, $u$ correctly computes $b_{u}$, proving (i); it can also immediately determine the feasibility of any of those links with respect to any pair of labels, and thus answer correctly any received query, proving (ii).

Induction step. Let the theorem hold for all nodes $x$ with $k-1 \geq h(x) \geq 0$; we will now show that it holds for $u$ with $h(u)=k$. By inductive hypothesis, it receives from each child $y$ the best feasible swap edge for each ancestor of $y$, including $u$ itself. Hence, based on these lists and on the locally available set InSwap $(u), u$ can correctly determine its optimal swap edge, as well as its best feasible swap edge for each of its ancestors.

The functioning of the Algorithm ALL BRIDGES-2 can be followed in the example of Figure 2.b and in particular through the convergecast, starting from the two leaves $G$ and $H$. After Phase $1, G$ and $H$ know their ancestors, namely: $F, D, C$. Node $G$ computes its bridge as $((G, D), 5)$, and the minimum swap edge for each of its ancestor, namely: $((G, D), 5)$ for $F,((G, D), 5)$ for $D$ and $((G, B), 7)$ for $C$, and sends these values to $F$. Similarly $H$ computes its bridge as $((H, E), 6)$, and the minimum swap edge for each ancestor, namely: $((H, E), 6)$ for $F,((H, E), 6)$ for $D$ and $N I L$ for $C$, and sends these values to $F$. $F$ computes its optimal swap edge as the minimum among its incident edges, that is, $((F, C), 4)$, the edge coming from $G$, $(G, D)$, having now distance 6 , and the edge coming from $H,(H, E)$, having now 
distance 7 . Hence it selects $((F, C), 4)$, as bridge and computes the minimum feasible swap edge for each of its ancestor, namely: $((F, C), 4)$ for $D$, and $((F, B), 5)$ for $C$, and sends these values to $F$. $D$ selects as bridge the edge coming from $F$, with distance incremented by 1 , that is $((F, C), 5)$, and sends $((F, B), 6)$ for $C$. $C$ can finally selects its bridge, considering the information coming from $D$ and that coming from $E$ which is $N I L$, as $((F, B), 7)$.

Let us now analyze the complexity of the algorithm:

\section{THEOREM 5}

The message complexity of Algorithm ALL BRIDGES-2 is exactly $2(n-1-\delta(r))$, where $\delta(r)$ is the degree of $r$.

Proof In the broadcast phase, every node except the root and its children receives a message. In the convergecast phase, every node except the root and its children sends a message.

\section{THEOREM 6 The data complexity of Algorithm ALL BRIDGES-2 is exactly $2 n_{r}^{*}$.}

Proof In the broadcast phase, every node (except the root and its children) receives the labels of all its ancestors. In the convergecast phase, every node (except the root and its children) sends a swap edge for each of its ancestors.

\section{Concluding Remarks}

In this paper we have presented simple and efficient distributed algorithms for computing the optimal swap edges of a shortest-path tree. One algorithm uses messages containing a constant amount of information, while the other is tailored for systems that allow long messages. Both algorithms exchange a quantity of information which is no more, and often significantly so, than that required to construct the shortest-path spanning-tree; also, they require information that can be acquired, with no increase in order of magnitude, during the shortest-path spanning-tree construction.

Among the possible development of this study, we mention that would be interesting to study how to recover from multiple link failures, following the same strategy of storing in the routing tables the information useful for finding alternative paths. The problem appears much more complex. In addition, the size of the routing tables is limited [9], hence the additional information to store in order to compute alternative paths in presence of faults must be also be small.

The proposed algorithms allow for the efficient construction of point-of-failure shortest-path rerouting service. To do so, the proposed computation must be carried out for the $\boldsymbol{n}$ shortest path trees, each having as root a different vertex of the graph. In this regards, an interesting open problem is whether it is possible to achieve the same goal in a more efficient way than by performing $\boldsymbol{n}$ independent computations. For example, it is known that the constructions of all-pairs shortest-paths can be done more efficiently than $\boldsymbol{n}$ independent constructions of a single shortest-path spanningtree (e.g., [1]); the research question is whether something similar holds also in this context. 


\section{Acknowledgments}

Part of this work was carried out while the the first and last authors were visited by the others at the University of Ottawa and at Carleton University. This work was supported in part by the Natural Sciences and Engineering Research Council of Canada, and by "Progetto ALINWEB: Algoritmica per Internet $\mathbf{e}$ per il Web", MIUR Programmi di Ricerca Scientifica di Rilevante Interesse Nazionale.

\section{References}

[1] Y. Afek, M. Ricklin Sparser: a paradigm for running distributed algorithms. Journal of Algorithms, 14:316-328, 1993.

[2] B. Awerbuch, R. Gallager A new distributed algorithm to find breadth first search trees. IEEE Transactions on Information Theory, 33:315-322, 1987.

[3] K. M. Chandy, J. Misra Distributed computation on graphs: shortest path algorithms. Communication of ACM, 25:833-837, 1982.

[4] G. N. Frederikson A distributed shortest path algorithm for planar networks. Information and Computation, 86:140-159, 1990.

[5] P. Humblet. Another adaptive distributed shortest path algorithm. IEEE/ACM Transactions on Communications, 39(6):995-1003, 1991.

[6] H. Ito, K. Iwama, Y. Okabe, T. Yoshihiro Polynomial-time computable backup tables for shortest-path routing. Proc. of 10th Colloquium on Structural Information and Communication Complexity (SIROCCO 2003), 163-177, 2003.

[7] P. Narvaez, K.Y. Siu, H.Y. Teng New dynamic algorithms for shortest path tree computation IEEE Transactions on Networking, 8:735-746, 2000.

[8] E. Nardelli, G. Proietti, P. Widmayer Swapping a failing edge of a single source shortest paths tree is good and fast. Algoritmica, 35:56-74, 2003.

[9] L. L. Peterson, B. S. Davie. Computer Networks: A Systems Approach, 3rd Edition. Morgan Kaufmann,2003.

[10] R. E.Tarjan Application of path compression on balanced trees. Journal of ACM, 26:690715, 1979. 